\title{
EBSD Performed “In-Situ” on a Dual-Beam FIB
}

\author{
J.K. Farrer, M.C. Chipman, and M. Tiner \\ TSL/EDAX, Draper, UT 84020
}

An electron-backscattered diffraction (EBSD) pattern is generated by electrons that are backscattered from the top 10-50 nm of the surface of a crystalline solid. Therefore, the surface of the solid must be carefully prepared to be free from damage to the atomic planes. It has been shown that a FIB can be used to prepare the surface of a material for EBSD analysis.[1] Many technologically important applications can benefit from the FIB as a sample preparation tool. However there are also many applications that require the material of interest to remain under vacuum, lest an oxide layer form on the surface and obscure the backscattered signal. For example, when exposed to atmosphere, nano-scale metallic interconnects, used in microelectronic devices, can oxidize rapidly, making them very difficult to analyze by EBSD.

One solution to this problem is having an EBSD detector installed on a dual-beam FIB, where both a focused-ion beam as well as an electron beam are available in the SEM chamber. The EBSD analysis can then be performed immediately after preparation with the ion beam and without breaking vacuum. This paper reports the use of an EBSD system installed on a dual-beam FIB (FEI Strata $\left.^{\mathrm{TM}} \mathrm{DB} 235\right)$. The design for this particular microscope required the use of a pre-tilted specimen holder $\left(45^{\circ}\right)$ in order to achieve the appropriate angle with the incident electron beam (EBSD systems require that the sample is tilted approximately $70^{\circ}$ with respect to the beam). It was shown that a sample mounted on the pre-tilted specimen holder can be cut and polished with the ion beam, in cross section or in plan view. The sample can then be rotated and tilted for EBSD analysis without remounting the specimen or breaking the chamber vacuum. The schematics shown in figure 1 represent the sample holder inside the dual-beam chamber positioned such that the ion beam is parallel to the top surface of the specimen (a) and then positioned for EBSD analysis of the same surface (b).

EBSD data from specimens analyzed using the dual-beam FIB/EBSD system were compared with data from specimens that were allowed to oxidize in atmosphere. The specimens analyzed were first polished using the ion beam. The settings and parameters of the EBSD detector and software were identical during the data collection for both specimens. Image quality maps of the respective samples are compared in figure 2. These maps are produced from EBSD patterns acquired from copper damascene lines deposited in amorphous $\mathrm{SiO}_{2}$. The quality (i.e. the degree of contrast and sharpness of the band edges) of the patterns, acquired at each point in a specified area on the surface, is measured and a grayscale value is assigned-white representing the highest measurable pattern quality and black the lowest.[2] The pattern quality, which affects the ability of the software to index the patterns and create maps based on crystallography, is seen to be much lower in the map from the oxidized sample. Figure 3 shows distinct grain maps corresponding to the image quality maps from the two specimens. Due to the increased pattern quality, the grain map from the specimen analyzed in the dual-beam FIB (a) more accurately represents the grain structure at the surface.

Further comparisons performed by changing the parameters of the ion beam and the degree of sample exposure to atmosphere will also be reported, as well as comparisons made with samples that were prepared using a broad-beam ion source.[3] 
[1] T.L. Matteson et al., J. Electron. Mater. 31 (2002) 33.

[2] B.L. Adams et al., Metall. Trans. A 24A (1993) 819

[3] M.M. Nowell, J. Electron. Mater. 31 (2002) 23.

[4] Use of the dual-beam FIB was supported by the director, Office of Science, Office of Basic Energy Sciences under the US Department of Energy DE-AC03-76SF00098.
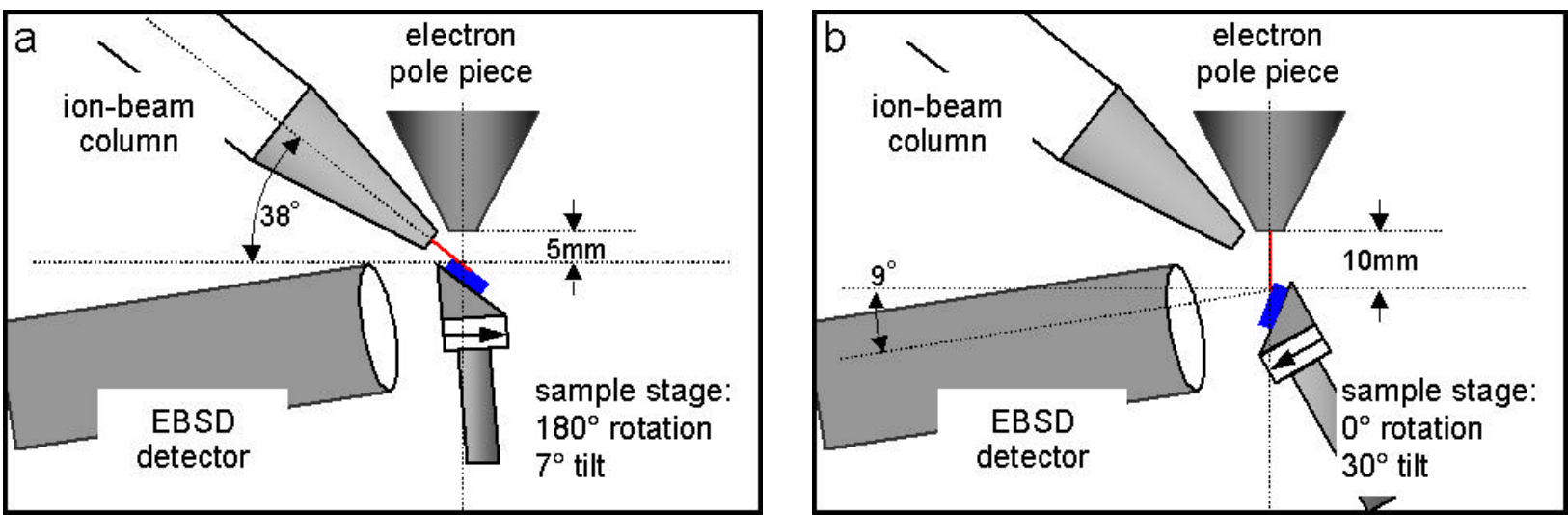

Fig. 1. Schematics representing the sample holder inside the dual-beam chamber positioned such that the ion beam is parallel to the top surface of the specimen (a) and then positioned for EBSD analysis of the same surface (b).
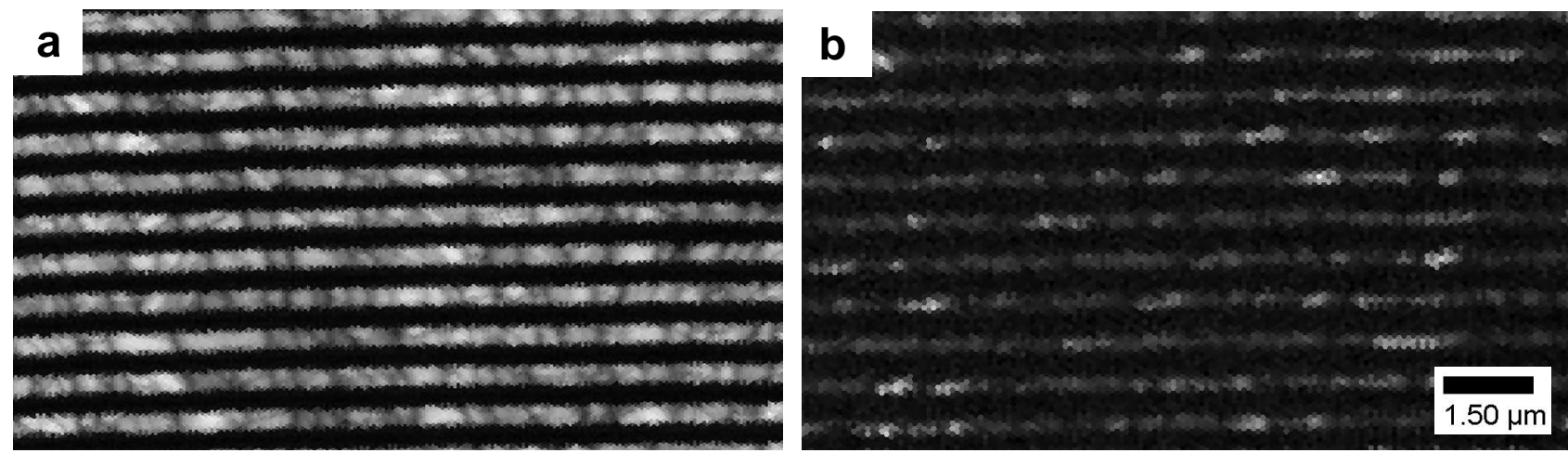

Fig. 2. Image quality maps from a specimen polished and analyzed using the dual-beam FIB/EBSD system (a) and from a specimen that was allowed to oxidize in atmosphere (b).
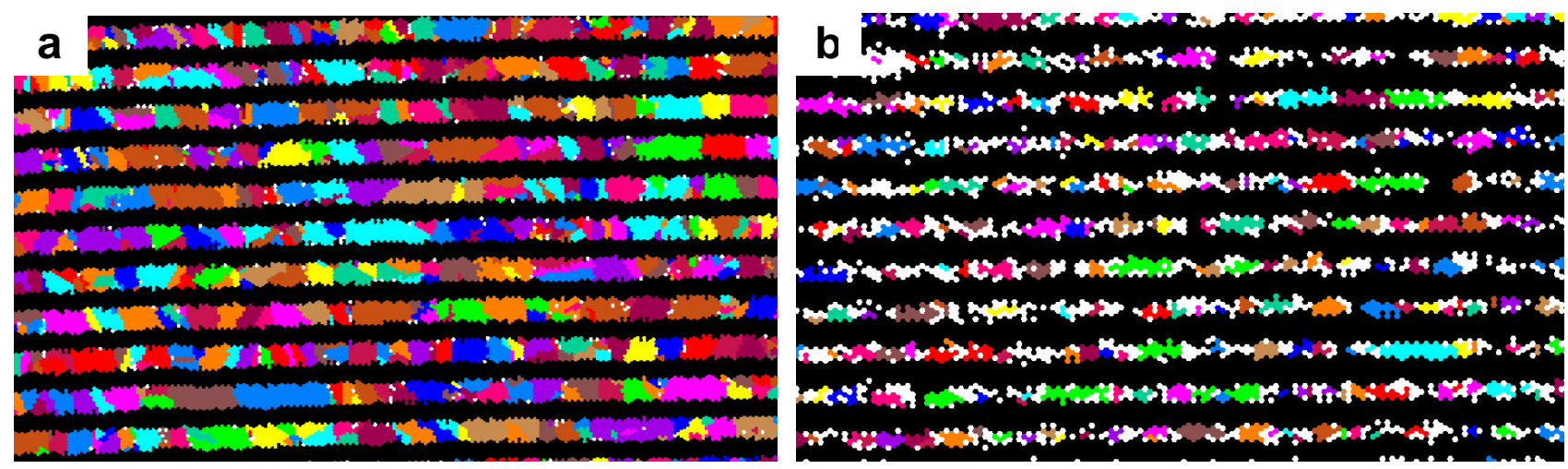

Fig. 3. Distinct grain maps corresponding to the image quality maps above. 